\title{
MCRO-ECP: Mutation Chemical Reaction Optimization based Energy Efficient Clustering Protocol for Wireless Sensor Networks
}

\author{
Ravuri Daniel $^{{ }^{*}}$ and Kuda Nageswara Rao ${ }^{2}$ \\ ${ }^{1}$ Department of Computer Science, Faculty of Technology, Debre Tabor University, Ethiopia \\ [danielravuri@gmail.com] \\ ${ }^{2}$ Department of Computer Science \& Systems Engineering, Andhra University College of Engineering (A), \\ Andhra University, Visakhapatnam, India \\ [knraoauce@andhrauniversity.edu.in] \\ *Corresponding author: Ravuri Daniel
}

Received August 31, 2018; revised October 30, 2018; accepted December 17, 2018; published July 31, 2019

\begin{abstract}
Wireless sensor networks encounter energy saving as a major issue as the sensor nodes having no rechargeable batteries and also the resources are limited. Clustering of sensors play a pivotal role in energy saving of the deployed sensor nodes. However, in the cluster based wireless sensor network, the cluster heads tend to consume more energy for additional functions such as reception of data, aggregation and transmission of the received data to the base station. So, careful selection of cluster head and formation of cluster plays vital role in energy conservation and enhancement of lifetime of the wireless sensor networks. This study proposes a new mutation chemical reaction optimization (MCRO) which is an algorithm based energy efficient clustering protocol termed as MCRO-ECP, for wireless sensor networks. The proposed protocol is extensively developed with effective methods such as potential energy function and molecular structure encoding for cluster head selection and cluster formation. While developing potential functions for energy conservation, the following parameters are taken into account: neighbor node distance, base station distance, ratio of energy, intra-cluster distance, and $\mathrm{CH}$ node degree to make the MCRO-ECP protocol to be potential energy conserver. The proposed protocol is studied extensively and tested elaborately on NS2.35 Simulator under various senarios like varying the number of sensor nodes and $\mathrm{CHs}$. A comparative study between the simulation results derived from the proposed MCRO-ECP protocol and the results of the already existing protocol, shows that MCRO-ECP protocol produces significantly better results in energy conservation, increase network life time, packets received by the BS and the convergence rate.
\end{abstract}

Keywords: Clustering, Wireless sensor network, Chemical reaction optimization, Mutation, Energy efficient. 


\section{Introduction}

The recent progress in Information Tech (IT) and Integrated Circuits (IC) fields has led to the development of low cost and compact sensor nodes. An integral of Internet of everything (IoE) is Wireless sensor network (WSN) and it enables data sharing amongst billions of devices, with higher user control. WSN comprises of large number of sensor nodes arranged on ad-hoc basis for observation and interaction with the physical world. Every sensor node comprises of four components: sensors, microcontrollers, power suppliers and transreceivers. The prime function of the sensors present in the sensing unit it to measure and caluculate the physical parameters from the real worls such as temperature, pressure, humidity, vibration, acoustic, infrared signals and vehicular movements, etc.,. The value mesured by the sensing unit is received and processed by the processing unit and then it is sent to the base station (BS) through either single-hop or multi-hop communication. WSNs are popularly used in tracking and monitoring applications that are usually deployed in areas like milatry surveillance, disaster management, agriculture, healthcare, automation industry, inventory control, etc., where the intervention of humans is not very feasible. The major challenge encountered in WSN design is utilization of energy, bandwidth and memory. To replace or recharge the batteries of the sensors becomes almost impossible because of the remoteness of the location. Also the cost of transmission almost exceeds the costs of the sensing and processing units in WSNs. Therefore, an effective protocol with an energy effecient data transmission needs to be developed to send data from sensor nodes to the respective BS to enhance the lifetime of the network. Extensive studies are carried out in clustering, as it is well known for optimization of energy conservation in wireless sensor networks $[1,2,3,4]$. In the process of optimization the organising of sensor nodes into groups are called as clusters. In the cluster a head is selected which is referred as the clusted head $(\mathrm{CH})$ and other snesor nodes are referred as cluster members (CM). The typical clustering architecture of WSNs is shown in Fig. 1. The two types of traffic in clustering are intra-cluster and inter-cluster. Intra-cluster traffic is the data transmission that happens within the cluster and Inter-cluster traffic is the data transmission that happens amongst the cluster. The role of the members in a cluster (CM) is to measure the real world parameters and send the measured values to their resapective $\mathrm{CH}$. Once the $\mathrm{CH}$ receives the data, it aggregates (evades redundant data) and transmits the data aggregated to the BS either directly or through CHs which get as intermediate. The entire process is systematic in which the cluster members transmit data to the $\mathrm{CH}$ and the $\mathrm{CH}$ transmits to the BS.

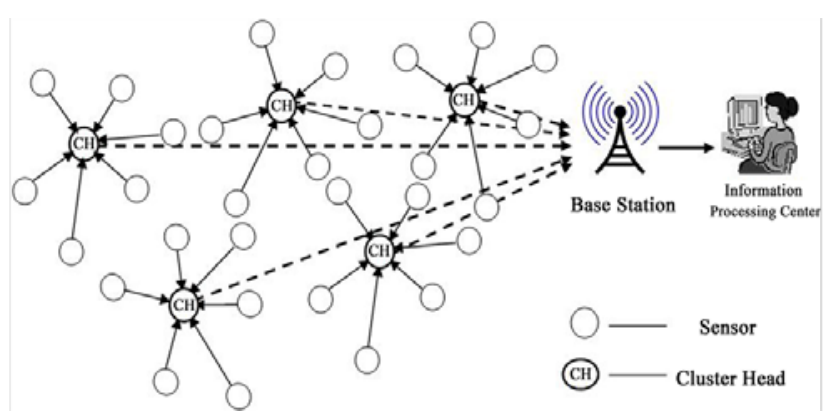

Fig. 1. Architecture of clustering in wireless sensor network.

Difficulty arises in choosing the $\mathrm{CH}$ which becoms an NP-hard problem, as the selection of ' $\mathrm{m}$ ' $\mathrm{CHs}$ among ' $\mathrm{n}$ ' sensor nodes provides ${ }^{\mathrm{n}} \mathrm{C}_{\mathrm{m}}$ possibilities. Also the computational complexity 
differs exponentially for the large scale networks. Likewise, during cluster formation, in a given ' $n$ ' sensor nodes and ' $m$ ' CHs, if each sensor node has ' $\mathrm{p}$ ' CHs as average in the communication range, then the valid number of assignments are $\mathrm{p}^{\mathrm{n}}$. The computational complexity changes exponentially, even here. Classical approaches are found wanting in resolving such type of situations.Various optimization approaches have been used to solve clustering problems in wireless sensor networks. Evolutionary algorithms, inspired by the biological evolution and social behaviour of organisms are the most powerful among optimization techniques. Genetic algorithms (GA) [5, 6] were developed based on the natural process of evolution. Particle swarm optimization (PSO) [7] was inspired by the social behavior of bird flocking. Ant colony optimization (ACO) [8] resembles PSO based on the capability of ants to find the shortest route between their nest and a food source. Artificial bee colony [9] was inspired by the echolocation behaviour of bees. Harmony search [10] was based on the natural musical performance of a musician searching for an optimal state of harmony. A recent study, new chemical reaction inspired evolutionary algorithm named "chemical reaction optimization (CRO)" [11] was sighted in literture. CRO is a low latency algorithm that represents the interaction of molecules in chemical reactions in order to reach a low energy stable state.

In wireless sensor networks, very few instances have been sighted using CRO, however, an effective version of CRO is the real-coded chemical reaction optimization (RCCRO) [12], which can process a large set of concurrent problems. HP-CRO [13], an algorithm based on PSO and CRO, produces more distinguished results compared to RCCRO. However, orthogonal chemical reaction optimization (OCRO) [14] algorithms have proved to be the best when compared with RCCRO and HP-CRO. OCRO is efficient in solving high-dimension functions but found wanting in respect of low-dimensional functions. Mutation chemical reaction optimization (MCRO) algorithm [15] was observed to be efficient in solving both the high and low dimensional functions. The convergence rate of MCRO is very high when compared with the traditional versions of CRO.

The proposed algorithm is based on a recently developed process for chemical reaction optimization (CRO) and two alteration operators like turning operator and mutation operator. Three types of mutation operators like uniform, nonuniform, and polynomial [16] were combined with chemical reaction optimization and turning operator to find the most appropriate framework. The best solution among these three options was selected to be a mutation chemical reaction optimization algorithm for global optimization. The current proposition is an energy efficient clustering based on mutation chemical reaction optimization (MCRO) algorithm termed MCRO-ECP. The proposed protocol combines CRO with a turning operator and a mutation operator. This meathod provides a solution to estimate the combined performance of the three types of mutation operators. (uniform, non-uniform and polynomial). The proposed MCRO-ECP could be a better choice for such NP-hard problems as it is convenient for implementation, high in quality of solution, feasible to escape from the local optima and quick in convergence.

This study proposes a new protocol with a combination of two phases, selection of the cluster head (CH) amongst the nodes and formation of the cluster using a single algorithm MCRO. This proposal is novel and almost first of its kind in WSNs. The first phase efficiently selects the optimum number of $\mathrm{CHs}$ and an effective location among the normal sensor nodes. Once the selection of optimal CHs is done the second phase progresses where the organization of cluster takes place. After the selection of optimal CHs, in the second phase- the cluster formation, member sensor nodes get assigned to the $\mathrm{CHs}$ based on the derived linear programming model as a cost function. MCRO-ECP is designed with potential molecular 
structure encoding schemes. Potential energy functions are developed considering parameters as those of neighbour node distance, base station distance, energy ratio, intra-cluster distance and $\mathrm{CH}$ node for energy efficy. The protocol is tested NS 2.35 realistic platform extensively to demonstrate its superiority over other existing algorithms. The features of MCRO-ECP are listed below.

- Adoption of Linear programming models for selection of CHs and cluster formation.

- Introduction of a new protocol that combines CRO with a turning operator and a mutation operator. This Enabling quick convergence and capability to break out from the local optima resulting in efficient data transmission and enhanced network lifetime.

The paper is presented in four sections. Section 2-agives review of related work. Section 3-the proposed approach of MCRO-ECP. Section 4-simulation results discussion. Section- 5 is conclusions and recommendations.

\section{Related Work}

In the recent years extensive studies have been carried out in the development of clustering protocols. for WSNs. This section details clustering protocols based on brute force and nature inspired approaches.

\subsection{Brute Force Approaches}

LEACH[17] is a popular distributed clustering protocol in which the $\mathrm{CH}$ gets elected on the basis of probability. The lacuna of this algorithm is that there is a chance of a $\mathrm{CH}$ with very low energy level to get elected, therefore the $\mathrm{CHs}$ will die quickly which will further degrade the performance of the network. Many improvements to LEACH $[18,19]$ and hierarchical protocols [20, 21] have been proposed, with emphasis on improved network life. Such improvements were found to be unsuitable for large size networks. Likewise LDC [22] and GLBCA [23] have also proved to be unsuitable in view of improper clusterformation and high execution times respectively.

\subsection{Nature Inspired Approaches}

Large number of clustering protocols are developed and proposed based on nature inspired approaches. Centralized leach (LEACH - C)[24] is developed and implemented with simulated annealing meathod. LEACH-C performs better than LEACH. Tillett et al., [25] have proposed a PSO approach to select the optimal location of CHs. However, it completely ignores the distance to the base station. Enan et al., [26] have presented an energy-aware evolutionary routing protocol (EAERP) for dynamic clustering. Even here possibilities of a node with low energy getting nominated exist. P.C.Srinivas Rao et al., [27] have proposed a PSO based cluster head selection protocol, which also does not consider residual energy levels of the sensor nodes during cluster formation. Latiff et al., [28] proposed an energy based CH selection using PSO-C by using diferent parameters such as ratio of total initial energy and average intra-cluster distance of all nodes to the total current energy of the all CHs. The main drawback of this algorithm is that, it assigns the non-cluster head nodes to the adjacent $\mathrm{CH}$ in the cluster formation phase, resulting in decreased network lifetime. Base station distance (which plays a very important role in reducing energy consumption, is not factored) in direct communication of CHs to BS. Buddha and Lobiyal [29] proposed a energy aware cluster head selection protocol using PSO which completely ignores the cluster arrangement stage. In [30], 
the proposed evolutionary method for load balanced algorithm. The limitation of this protocol is selected the cluster heads randomly, which causes energy inefficiency of the network. Kuila P. et.al [31], proposed differential evolution protocol for clustering in WSNs. Here too the base station distance is ignored in cluster formation and the CHs are selected randomly, which may lead to energy depletion. P.C.Srinivasa Rao et al., [32] proposed an energy efficient clustering protocol based on chemical reaction optimization approach.

\section{Proposed Approach: MCRO-ECP}

First time in WSN, a mutation chemical reaction optimization (MCRO) based cluster head selection and cluster formation is proposed. The operation is divided into two sections the setup phase and the steady state phase. Each round of communication commences with a setup phase (when the clusters are organized by MCRO), followed by a steady state phase (when CMs and CHs communicate) using TDMA protocol. Aggregated packets at the level of $\mathrm{CH}$ are routed through intermediate $\mathrm{CHs}$ to the BS using CSMA/CA protocol.

A mutation chemical reaction optimization (MCRO), which uses CRO and two adjustment operators, turning operator and mutation operator, generates various molecular structures to produce high quality results and accelerate convergence. Details of turning operator and the mutation operator are detailed as follws.

\subsection{Turning Operator}

Turning operator is a new operator that is merged into a sub algorithm named neighborhood search operator. Neighborhood search operator is used in three types of elementary reactions:

1. Intermolecular ineffective collision,

2. On-wall ineffective collision, and

3. Decomposition

to transform the molecular structure from the neighborhood of the operand [33]. The new molecular structures of the solution are calculated as Equation (1) and turning operator is generated once for an objective function $\left(f_{i}\right)$ by using Equation (2). This new operator can improve the optimal quality and reliability of the algorithm:

$$
\left.\mathrm{nS}=(\mathrm{pS}+\mathrm{r})^{*} \mathrm{~s}\right) * \mathrm{~T}\left(\mathrm{f}_{\mathrm{i}}\right)
$$

where, $\mathrm{nS}$ is new Structure, $\mathrm{pS}$ is previous Structure, $\mathrm{r}$ is Random Gaussian, $\mathrm{s}$ is the stepSize, and $\mathrm{T}$ is the turning Operator.

$$
\mathrm{T}\left(f_{\mathrm{i}}\right)=\left\{\text { random interval }\left[1, \mathrm{e}^{-300}\right]\right\},(1 \leq i \leq 23)
$$

\subsection{Mutation Operator}

Mutation operator is migrated into MCRO to improve solution diversity and accelerate convergence by generating various molecular structures. These structures are generated by using the processing mutation formula. This action increases the chance of finding the global optimal solution and avoids being trapped into a local optimal solution; therefore,-accelerates convergence. In our research, the best mutation operator that matches our algorithm was selected the mutation operators (uniform, nonuniform, and polynomial). Uniform and non-uniform mutations are presented in basic GAs and their extending development of algorithm [5]. Polynomial mutation is a popular one that was first introduced in non-dominated sorting genetic algorithm (NSGA) and NSGA-II [6]. Uniform, non-uniform, and polynomial mutations are calculated as equation (3), (4), and (5), respectively. Input data for mutation operator algorithm is a set of molecular structures presented as $v=\left\{v_{1}, v_{2}, \ldots\right.$, $\left.v_{\mathrm{v}}\right\}$, where $\mathrm{v}$ is the number of moles, $v_{i}$ is a member of $v, 1 \leq i \leq \mathrm{v}$. Likewise output data is a set 
of molecular structures presented as v́. The variable names such as $v$ and $v$ maybe present differently when they appear in other algorithms such as temp $p_{v}$, temp $p_{v}$, temp $p_{v 1}$, and temp $p_{v 2}$ :

$$
\begin{gathered}
\mathrm{u}_{\mathrm{i}}^{\prime}=[(\mathrm{r}[0-1]-0.5) \times \mathrm{p})+\mathrm{u}_{\mathrm{i}} \\
\mathrm{u}_{\mathrm{i}}^{\prime}=\left[\left(\mathrm{ul}-\mathrm{u}_{\mathrm{i}}\right)^{*}\left(1.0 \times \mathrm{r}[0-1]^{1.0-\text { FE } / \text { FE Limit })}{ }^{\mathrm{p}}\right]+\mathrm{u}_{\mathrm{i}}\right.
\end{gathered}
$$

where,

$p$ is perturbation,

$$
\begin{aligned}
& u l=\left\{\begin{array}{l}
U, r[0-1]<0.5 \\
L, r[0-1] \geq 0.5
\end{array}\right. \\
& \mathrm{u}_{\mathrm{i}}^{\prime}=\mathrm{u}_{\mathrm{i}}^{\prime}+\Delta \mathrm{q}^{2} \times \Delta \mathrm{q} \begin{cases}\left(2.0 \times \mathrm{r}+(1.0-2.0 ?) \times(1.0-\Delta 1)^{\mathrm{D}}\right)(1.0 /(\mathrm{D}+1.0) & \mathrm{r}:[0-1]<0.5 \\
1.0-\left(2.0 \times(1.0-\mathrm{r})+2.0 \times(\mathrm{r}-0.5) \times(1.0-\Delta 2)^{(\mathrm{D}+1.0)}\right)(1.0 /(\mathrm{D}+1.0)) & \mathrm{r}:[0-1] \geq 0.5\end{cases}
\end{aligned}
$$

where, $\Delta 1=\frac{u_{i}^{\prime}-\mathrm{L}}{\mathrm{d}(\mathrm{U} \text { and } \mathrm{L})}, \Delta 2=\frac{\mathrm{U}-u_{\mathrm{i}}^{\prime}}{\mathrm{d}(\mathrm{U} \text { and } \mathrm{L})}, \mathrm{r}$ is the random number, $\mathrm{U}$ is the upper bound, and $\mathrm{L}$ is the lower bound.

Mutation operator is applied at the initilization stages of the applied algorithm. During the iteration phase, the mutation operator is applied to process all types of basic reactions such as, on-wall ineffecient collision, intermolecular ineffective collision, decomposition and synthesis to bring about a changed molecular structure.

Mutation is a principal component to improve the performance of MCRO, as it can spread the search space by randomly sampling new points. Furthermore this mutation operator algorithm increases the chance of generating more powerful result not less than twice of original CRO for every basic reaction. There are the four elementary reactions's explained in algorithm-1. The proposed algorithm, MCRO for $\mathrm{CH}$ selection and cluster formation approaches are as shown in algorithm-1.

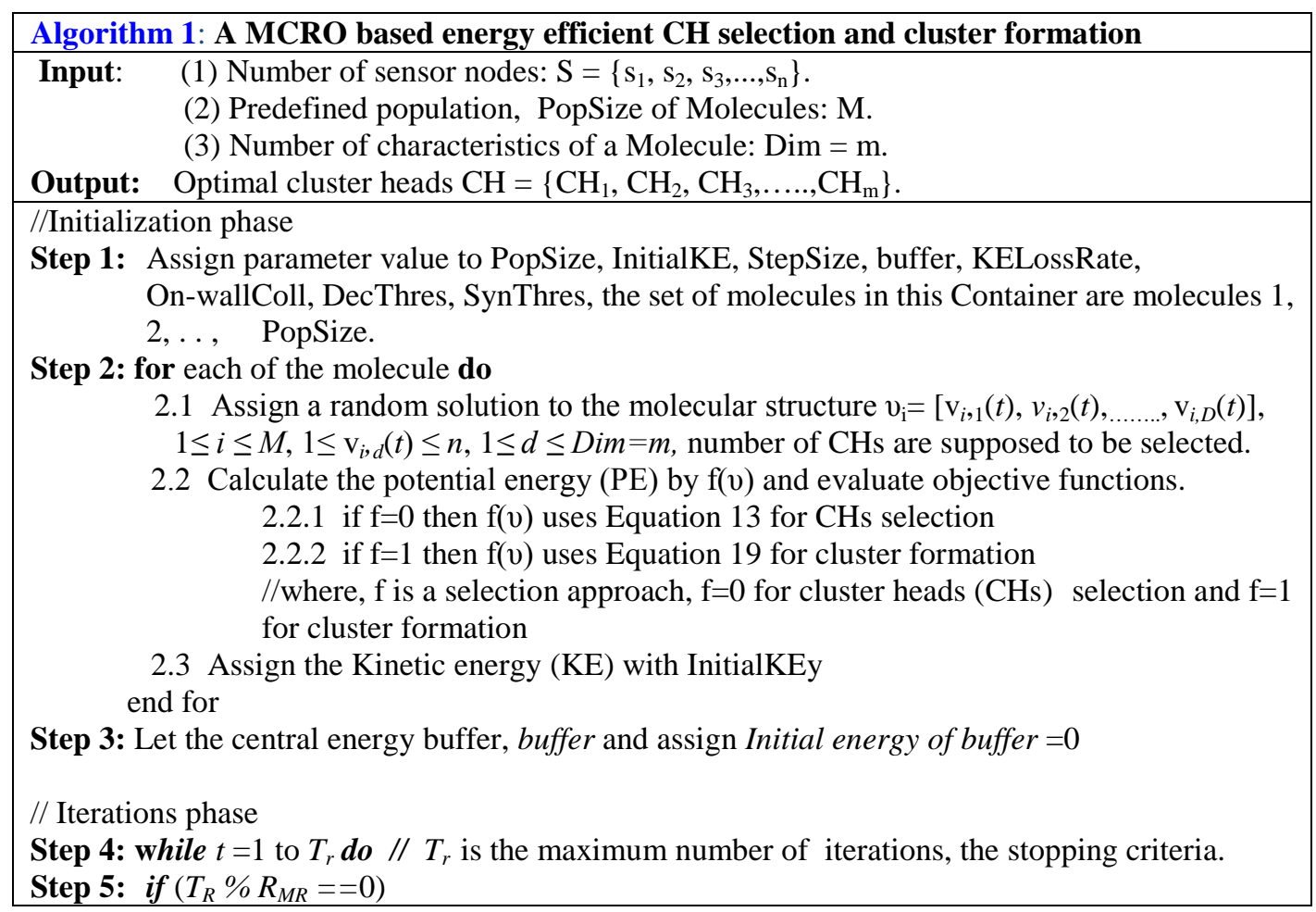




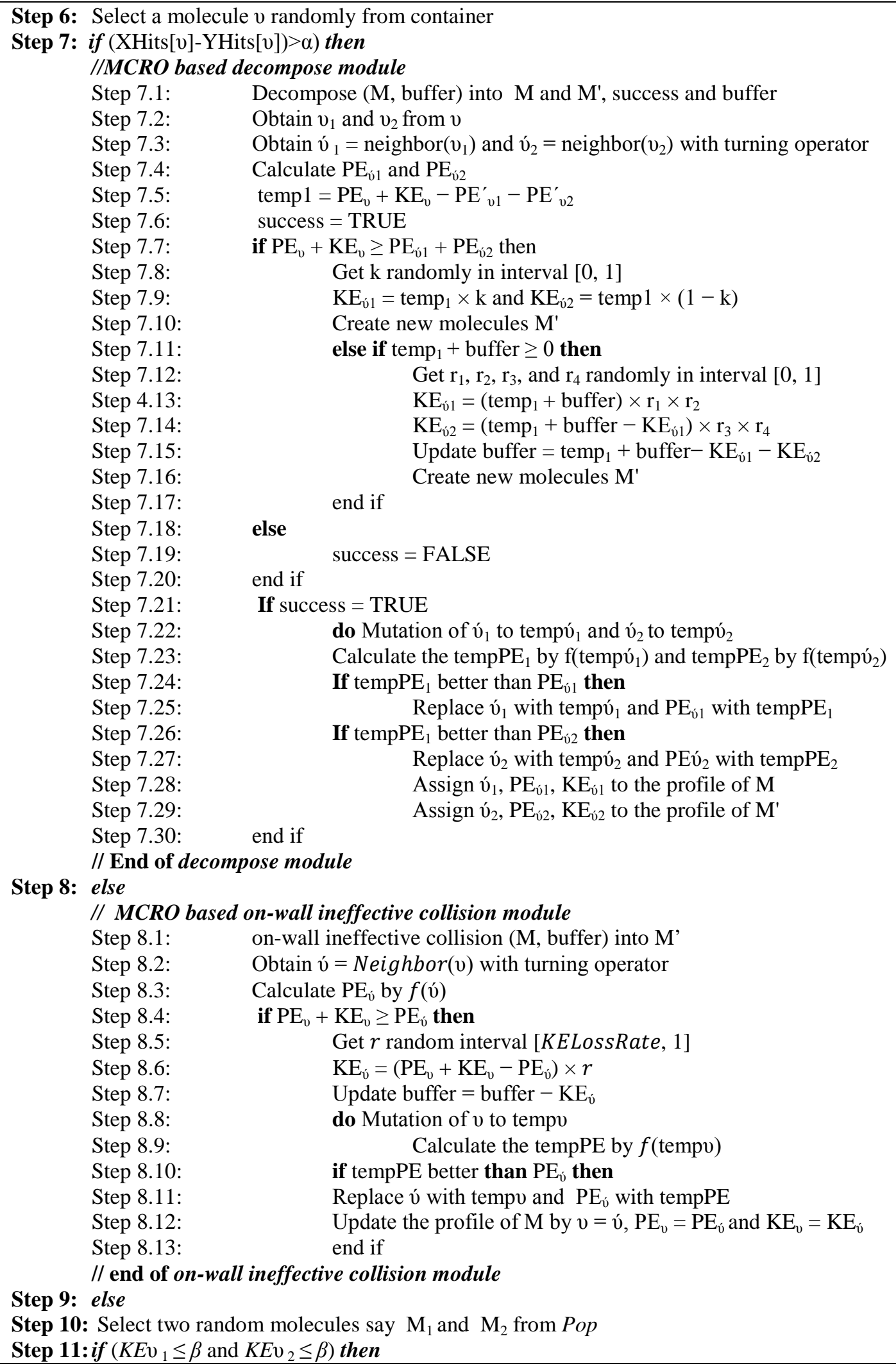




\section{// MCRO based synthesis module}

Step 11.1: Synthesis $\left(\mathrm{M}_{1}, \mathrm{M}_{2}\right)$ into $\mathrm{M}^{\prime}$

// satisfies the energy conservation condition

Step 11.2: Obtain $v_{1}$ from $v_{1}$ and $v_{2}=v_{2}$

Step 11.3: Calculate $\mathrm{PE}_{v 1}$

Step 11.4: if $\mathrm{PE}_{v 1}+\mathrm{PE}_{\hat{v} 2}+\mathrm{KE}_{v 1}+\mathrm{KE}_{\hat{v} 2} \geq \mathrm{PE}_{\hat{v} 1}$ then

Step 11.5: $\quad$ success $=$ TRUE

Step 11.6: $\quad \mathrm{KE}_{v^{\prime} 1}=\mathrm{PE}_{\mathrm{v} 1}+\mathrm{PE}_{\mathrm{v} 2}+\mathrm{KE}_{\mathrm{v} 1}+\mathrm{KE}_{\mathrm{v} 2}-\mathrm{PE}_{\mathrm{v} 1}$

Step 11.7:

Step 11.8:

do Mutation of $v_{1}$ to tempv $v_{1}$

Step 11.9:

Step 11.10:

Step 11.11:

Step 11.12:

Step 11.13: else

Step 11.14:

Calculate the tempPE by $\mathrm{f}\left(\right.$ tempv $\left._{1}\right)$

If tempPE better than $\mathrm{PE}_{\tilde{v} 1}$ then

Replace $v_{1}$ with temp $v_{1}$ and $\mathrm{PE}_{v 1}$ with tempPE

Calculate the tempPE $E_{1}$ by $\mathrm{f}\left(\right.$ tempv $\left._{1}\right)$ and tempPE $\mathrm{C}_{2}$ by $\mathrm{f}\left(\right.$ tempv $\left._{2}\right)$ Assign $v_{1}, \mathrm{PEv}_{1}{ }_{1}$, and $\mathrm{KEv}_{1}{ }_{1}$ to the profile of $\mathrm{M}_{1}$

Step 11.15: end if

// end of synthesis module

Step 12: $\quad$ end if

Step 13: else

// MCRO based inter-molecular ineffective collision module

Step 13.1: Inter-molecular ineffective collision ( $\left.\mathrm{M}_{1}, \mathrm{M}_{2}\right)$ into $\mathrm{M}_{1}$ ' and $\mathrm{M}_{2}$ '

Step 13.2: Obtain $v_{1}=\operatorname{Neighbor}\left(v_{1}\right)$ and $v_{2}=\operatorname{Neighbor}\left(v_{2}\right)$ with turning operator

Step 13.3: Calculate $\mathrm{PE}_{\mathfrak{v} 1}$ and $\mathrm{PE}_{\hat{v} 2}$

Step 13.4: temp2 $=\left(\mathrm{PE}_{\hat{v} 1}+\mathrm{PE}_{\hat{v} 2}+\mathrm{KE}_{\hat{v} 1}+\mathrm{KE}_{\hat{v} 2}\right)-\left(\mathrm{PE}_{\hat{v} 1}+\mathrm{PE}_{\hat{v} 2}\right)$

Step 13.5: if $\mathrm{PE}_{\hat{v} 1}+\mathrm{PE}_{\hat{v} 2}+\mathrm{KE}_{\hat{v} 1}+\mathrm{KE}_{\hat{v} 2} \geq \mathrm{PE}_{\hat{v} 1}+\mathrm{PE}_{\hat{v} 2}$ then

Step 13.6: $\quad$ Get $\mathrm{p}$ randomly in interval $[0,1]$

Step 13.7: $\quad \mathrm{KE}_{\hat{v} 1}=$ temp $_{2} \times \mathrm{p}$ and $\mathrm{KE}_{\hat{v} 2}=\operatorname{temp}_{2} \times(1-\mathrm{p})$

Step 13.8: end if

Step 13.9: do Mutation of $v_{1}$ to tempv $v_{1}$ and $v_{2}$ to tempv $v_{2}$

Step 13.10: $\quad$ Calculate the tempPE $E_{1}$ by $f\left(\right.$ tempv $\left._{1}\right)$ and tempPE $E_{2}$ by $f\left(\right.$ tempv $\left._{2}\right)$

Step 13.11: If tempPE $E_{1}$ better than $\mathrm{PE}_{v 1}$ then

Step 13.12: $\quad$ Replace $v_{1}$ with tempv $v_{1}$ and $\mathrm{PE}_{v 1}$ with tempPE

Step 13.13: If tempPE $E_{2}$ better than $\mathrm{PE}_{\hat{v} 2}$ then

Step 13.14: $\quad$ Replace $v_{2}$ with tempv $v_{2}$ and $\mathrm{PE} v_{2}$ with tempPE

Step 13.15: Update the profile of $\mathrm{M}_{1}$ by $v_{1}=v_{1}, \mathrm{PE}_{\hat{v} 1}=\mathrm{PE}_{\dot{v} 1}$ and $\mathrm{KE}_{v 1}=\mathrm{KE}_{v 1}$

Step 13.16: Update the profile of $\mathrm{M}_{2}$ by $v_{2}=v_{2}$, $\mathrm{PEv}_{2}=\mathrm{PEv}_{2}$ and $\mathrm{KEv}_{2}=\mathrm{KEv}_{2}$

$/ /$ end of inter-molecular ineffective collision module

Step 14: Find for any new optimal minimum value

Step 15: end while

Step 16: Stop

\subsection{MCRO based Cluster Head Selection}

The Proposed protocol is designed with a molecular structure and a new energy function that could effectively and efficiently aid in calling out the $\mathrm{CH}$ and built potential clusters.

\section{A) Problem Formulation for $\mathrm{CH}$ Selection}

The proposed protocol is developed with an objective to perform optimal $\mathrm{CH}$ selection which would aid to enhance the network life span. To accomplish the objectivethe intra-cluster distance, minimum distance of base stationand maximum residual energy of the nodes are taken into account. Let us consider the function of minimum distance between the sensor nodes as $f_{1}$. To optimize the $\mathrm{CH}$ selection the $\mathrm{f}_{1}$ is to be minimized. Let $\mathrm{f}_{2}$ be the function of 
minimum base station distance and $f_{3}$ be the function be the function of energy ratio, i.e., the ratio of the energy consumed by the sensor nodes and the remaining energy of the sensor nodes. This ratio should be minimized to acheive optimal $\mathrm{CH}$ selection. All the three objectives could be accomplished, if the function range is maintained between 0 and 1 , which would result in optimal CH selection. The linear programming design of the optimal $\mathrm{CH}$ selection problem is given below:

$$
\begin{aligned}
& \text { Minimize } \mathrm{F}=\mathrm{f}_{1} \times \alpha_{1}+\mathrm{f}_{2} \times \alpha_{2}+\mathrm{f}_{3} \times \alpha_{3} \quad \text { Subject to } \\
& \mathrm{d}\left(\mathrm{s}_{\mathrm{i}}, \mathrm{CH}_{\mathrm{j}}\right) \leq \mathrm{d}_{\max }, \forall \mathrm{s}_{\mathrm{i}} \in \mathrm{S} \text {, and } \mathrm{CH}_{\mathrm{j}} \in \mathrm{C} \\
& \mathrm{E}_{\mathrm{CH}_{\mathrm{j}}}>\mathrm{T}_{\mathrm{h}}, 1 \leq \mathrm{j} \leq \mathrm{m} \\
& \alpha_{1}+\alpha_{2}+\alpha_{3}=1, \alpha_{1}, \alpha_{2} \text {, and } \alpha_{3} \in(0,1)
\end{aligned}
$$

The constraint (6) depicts that the communication range of the sensor node $\mathrm{S}_{\mathrm{i}}$ with $\mathrm{CH}_{\mathrm{j}}$ is maximum. The constraint (7) states that the energy of $\mathrm{CH}_{\mathrm{j}}$ nodes must be greater than the threshold energy $T_{h}$. In the constraint (8), $\alpha_{1}, \alpha_{2}$ and $\alpha_{3}$ are the control parameters of the function $f_{1}, f_{2}$ and $f_{3}$ respectively. It also ensures that those values must not be of 0 or $100 \%$ weight.

\section{B) Potential Energy Function for $\mathrm{CH}$ Selection}

The derivation of proposed potential energy function depends on the given parameters:

a) Neighbour node distance: We need to select the CHs such that distance from its neighbour nodes is minimal. In the intra-cluster communication process, sensor nodes consume some energy to communicate data to the CHs. If we reduce the neighbour node distance, energy of the intra-cluster communication also reduces.

$$
\text { Objective 1: } \text { Minimize }_{\mathrm{f}_{1}}=\sum_{\mathrm{j}=1}^{\mathrm{m}} \frac{1}{\mathrm{n}_{\mathrm{j}}}\left(\sum_{\mathrm{i}=1}^{\mathrm{I}_{j}} \mathrm{~d}\left(\mathrm{CH}_{\mathrm{j}}, \mathrm{s}_{\mathrm{i}}\right)\right)
$$

b) The distance of Base Station: It is the distance between a cluster head $\mathrm{CH}_{j}$ and the base station (BS). BS distance has a major role in the selection of energy efficient CHs.

$$
\text { Objective 2: Minimize } \mathrm{f}_{2}=\sum_{\mathrm{j}=1}^{\mathrm{m}} \frac{1}{\mathrm{~m}}\left(\mathrm{~d}\left(\mathrm{CH}_{\mathrm{j}}, \mathrm{BS}\right)\right)
$$

c) Energy ratio: It is the ratio of the energy consumed by the CHs and the residual energy of the CHs. When a $\mathrm{CH}$ consumes minimal energy during sensing, aggregating and transmitting then the residual energy if the $\mathrm{CH}$ will be higher therefore the $\mathrm{CH}$ will have lower energy ratio. Lower the $\mathrm{CH}$ energy ratio the greater is the chance of optimal selection of CHs.

$$
\text { Objective 3: Minimize } \mathrm{f}_{3}=\sum_{\mathrm{j}=1}^{m} \frac{\mathrm{E}_{\mathrm{C}}\left(\mathrm{CH}_{\mathrm{j}}\right)}{\mathrm{E}_{\mathrm{R}}\left(\mathrm{CH}_{\mathrm{j}}\right)}
$$

It is recommended to minimize the linear combination of all the above three functions together instead of minimizing each function seperately. MCRO-ECP adopts the following potential energy function.

$$
\text { Potential energy function } \mathrm{F}=\mathrm{f}_{1} \times \alpha_{1}+\mathrm{f}_{2} \times \alpha_{2}+\mathrm{f}_{3} \times \alpha_{3}
$$

where, $\alpha_{1}+\alpha_{2}+\alpha_{3}=1, \alpha_{2} \geq\left(\alpha_{1}+\alpha_{3}\right)$, also $0<f_{1}, f_{2}, f_{3}<1$

The prime objective of the proposal is to minimize the potential energy function. The lower the value of $P E$, the better is the stability of the molecule, i.e., the better is the $\mathrm{CH}$ selection. 


\subsection{Proposed MCRO based Cluster Formation}

After the selection of optimal CHs, with the derived potential energy function, the cluster formation algorithm is runned at the BS for the optimal assignment of the non-cluster head sensor nodes to the CHs.

\section{A) Linear Programming Model for Cluster Formation}

In the proposed cluster formation problem, the central objective is to increase the life time of the network by decreasing the energy consumption of the network. Let $\mathrm{g}_{1}$ be the function of a sensor node to the $\mathrm{CH}$ that would maximize the residual energy which would result in optimal cluster formation. Let $\mathrm{g}_{2}$ be the function to minimize the distance between a sensor node and $\mathrm{CH}$ and $\mathrm{CH}$ and BS. These two objectives function values are normalised between the range of 0 and 1 thus the lenier combinations of these two functions is minimized in an effecient manner. Let $\mathrm{a}_{\mathrm{ij}}$ be a Boolean variable defined by

$$
\begin{aligned}
& a_{i j}=\left\{\begin{array}{l}
1 \text { if } S_{i} \text { is assigned to } C_{j}, \forall_{i}, j: 1 \leq i \leq n, 1 \leq j \leq m \\
0 \text { otherwise }
\end{array}\right. \\
& \text { Minimize } G=g_{1} \times \beta_{1}+g_{2} \times \beta_{2} \text { subject to } \\
& \mathrm{d}\left(\mathrm{s}_{\mathrm{i}}, \mathrm{CH}_{\mathrm{j}}\right) \times \mathrm{a}_{\mathrm{ij}} \leq \mathrm{d}_{\text {max }}, \forall \mathrm{s}_{\mathrm{i}} \in \mathrm{S} \text { and } \mathrm{CH}_{\mathrm{j}} \in \mathrm{C} \\
& \sum_{\mathrm{j}=1}^{\mathrm{m}} \mathrm{a}_{\mathrm{ij}}=1
\end{aligned}
$$

The constraint (14) denotes that when the sensor node $\mathrm{s}_{\mathbf{i}}$ is in the maximum communication range it can be assignes to the $\mathrm{CH}_{\mathrm{j}}$ and constraint (15) denotes that the degree of $\mathrm{CH}_{\mathrm{j}}$ and $\mathrm{s}_{\mathrm{i}}$ can be assigned to only one $\mathrm{CH}_{\mathrm{j}}$.

\section{B) Potential Energy Function for Cluster Formation}

The potential energy derivation of function depends on the following parameters:

a) Intra-cluster distance: During the intra-cluster communication process, sensor nodes consume energy to transmit data to the CHs. So, when the distance in the intra-cluster is minimized it results in decreased energy consumption. To acheive this the CHs are selected with minimal distance from it's neighbour nodes.

$$
\text { Objective 1: Minimize } \mathrm{g}_{1}=\sum_{\mathrm{j}=1}^{\mathrm{m}}\left(\sum_{\mathrm{i}=1}^{\mathrm{l}_{\mathrm{j}}} \mathrm{d}\left(\mathrm{s}_{\mathrm{i}}, \mathrm{CH}_{\mathrm{j}}\right)\right)
$$

b) $\mathrm{CH}$ node degree: A sensor node $s_{i}$ should be paired with a $\mathrm{CH}_{j}$ which has lower node degree rather than any other $\mathrm{CH}$ in the communication range of the sensor node.

$$
\text { Objective 2: Minimize } \mathrm{g}_{2}=\frac{1}{\text { nodedegree }\left(\mathrm{CH}_{\mathrm{j}}\right)}
$$

The weight summation approach is utilized to minimize the energy consumptions of the two objective functions together instead of minimizing the functions seperately as these two are weably conflicting each other which is tackeled by a unique optimal solution. Therefore the following potential energy function is utilized.

$$
\text { Potential energy function: } \mathrm{G}=\mathrm{g}_{1} \times \beta_{1}+\mathrm{g}_{2} \times \beta_{2}
$$

where, $\beta_{1}+\beta_{2}=1$ 
The objective is to minimize the potential energy consumption. The lower the value of PE, better is the stability of the molecule, i.e., optimal assignment of non-CH sensor nodes to $\mathrm{CHs}$.

\section{Simulation Results and Performance Analysis}

The experimental simulation and performance analysis of proposed protocol, MCRO-ECP are discussed in this section. The energy model used in proposed work is described in [17]. All sensors are deployed randomly in the target area and can calculate distance to other sensors based on RSSI model[34]. The sensor nodes therefore do not require global position system. The sensors are taken into consideration to be stationary after operation and sensors are able to operate in $\mathrm{CH}$ and sensor node mode. All the sensors sense the data periodically and the assumption is that, they have data to communicate to cluster head or base station. The sensing and data transmission distances are in a circular field in which all the sensor nodes are uniform and pocess equal ability for implementation and communication. The communication links become wireless and symmetric and become established between the nodes when they are in the range of each other for communication. Life span of the network can be described in many ways [35].

The performance of the proposed protocol was investigated against the well known clustering protocols PSO-C [28], LDC [22], GLBCA [23], GALBCA [30], DECA [31] and CRO-ECA [32]. In order to provide a fair comparison, all the competent protocols along with the proposed protocol were implemented under the NS2.35 simulator. Detailed configuration of the wireless sensor network and the performance of the proposed protocol as against to existing protocols under varied performance metrics are disccused in the following sections.

\subsection{Simulation Setup}

In our experiment,variable number of sensor nodes 200, 400, 600, 800, and 1000 were deployed randomly in the area of $400 \times 400 \mathrm{~m}^{2}$. The cluster heads (CHs) were selected randomly as $10 \%$ of sensor nodes and base station was placed in the corner of the field, $(0,0)$. The values used in the first order radio model [17] are described in Table 1. The initial energy $\left(E_{\text {init }}\right)$ of a node is set to 2 joules, circuite energy $\left(E_{\text {elec }}\right)$ is 50 nano joules per bit, amplification of the free space energy $\left(\varepsilon_{\mathrm{fs}}\right)$ is set to $10 \mathrm{pJ} / \mathrm{bit} / \mathrm{m}^{2}$, amplification of the multipath transmission energy $\left(\varepsilon_{\mathrm{mp}}\right)$ is $0.0013 \mathrm{pJ} / \mathrm{bit} / \mathrm{m}^{4}$, maximum threshold distance $\left(\mathrm{d}_{\max }\right)$ is 120 metres, minimum threshold distance $\left(\mathrm{d}_{0}\right)$ is 87.7 metres, packet length is 512 bytes, and message size is 500 bits.

Table 1. Simulation setup of proposed MCRO-ECP.

\begin{tabular}{ll}
\hline \multicolumn{1}{c}{ Parameter } & \multicolumn{1}{c}{ Value } \\
\hline Sensor field area & $400 \times 400 \mathrm{~m}^{2}$ \\
Position of base station & $(0,0)$ \\
Number of sensors & $200,400,600,800,1000$ \\
Number of CHs & $10 \%$ of sensor nodes \\
Initial energy of sensor, $E_{\text {init }}$ & $2 \mathrm{Joules}$ \\
Circuite energy, $\mathrm{E}_{\text {elec }}$ & $50 \mathrm{~nJ} / \mathrm{bit}$ \\
Amplification of the free space energy, $\varepsilon_{f s}$ & $10 \mathrm{p} \mathrm{J} / \mathrm{bit} / \mathrm{m}^{2}$ \\
Amplification of the multipath Transmission energy $\varepsilon_{m p}$ & $0.0013 \mathrm{pJ} / \mathrm{bit} / \mathrm{m}^{4}$ \\
Maximum threshold distance, $d_{\max }$ & $120 \mathrm{mts}$ \\
Minimum threshold distance, $d_{0}$ & $87.7 \mathrm{mts}$ \\
Packet length & $512 \mathrm{bytes}$ \\
Message size & $500 \mathrm{bits}$ \\
\hline
\end{tabular}




\subsection{Simulation Parameters}

The following performance metrics were considered for performance evaluation of the MCRO-ECP.

A. Energy Consumption: The amount of energy consumed in joules in the network over a number of rounds, in each of which the energy utilised by the CHs for data collection, aggregation and transmission to the BS is calculated.

B. Network Lifetime: The life time of the network is defined here as the as death of first node (FND). The increase in the lifetime of the network proportionately enhances the performance of the network.

C. Packets received by BS: The total number of packets received by the BS over the span of network lifetime.

D. Convergence rate: To converge the global optimal solution, an algorithm performs certain number of rounds called as convergence rate.

\section{A) Performance of Energy Consumption}

The total energy consumption of the network over number of rounds, in each round, CHs collect data, aggregate and route it to the BS. The unit measurement of energy consumption is joules. In the proposed protocol the performance of the energy consumption is tested under different scenarios by changing the number of sensor nodes and cluster heads. Here, the proposed protocol results are shown in conditions of the total energy consumption of the networks of 400 sensor nodes with 40CHs. Fig. 2 shows the comparitive results of the proposed protocol with the standard protocols. The proposed protocol outperforms the existing protocols on total energy consumption which is mesuered in joules. MCRO-ECP outperforms PSO-C by $27.1 \%$, LDC by $23.5 \%$, GLBCA by $20.6 \%$, GALBCA by $16.20 \%$ DECA by $11.8 \%$, and CRO-ECA by $7 \%$.

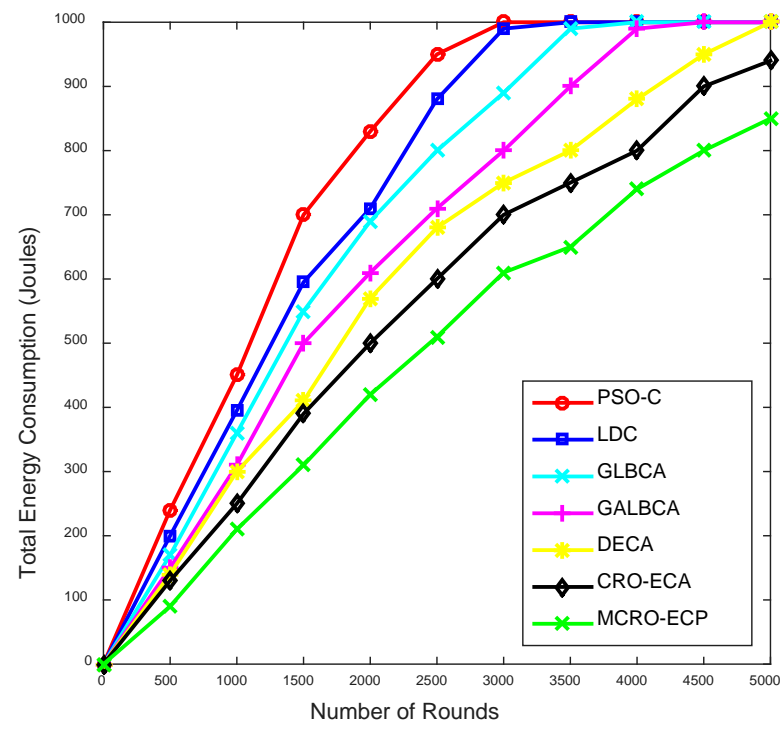

Fig. 2. Performance analysis of energy consumption in 400 sensor nodes and 40 cluster heads wireless sensor network.

\section{B) Analysis of Performance of Network Lifetime}

The life time of the network can be defined as the as death of first node (FND). As lifetime increases the network performance gets better. Fig. 3 shows the FND data of MCRO-ECP 
outperforms than CRO-ECA by $4.6 \%$, DECA by $9.6 \%$, GALBCA by $13.2 \%$, GLBCA by $19.3 \%$, LDC by $20.1 \%$, and PSO-C by $30.04 \%$.

\section{C) Analysis of Performance of Packets Receipt by BS}

The total number of packets transmitted to the BS in a span of network lifetime is another metric. The unit measurement of packets received by BS is packets/second. Fig. 4 shows the performance of packets received by base station which is measured by number of packets recieved by BS per second. MCRO-ECP outperforms than CRO-ECA by $4.8 \%$, DECA by $10.4 \%$, GALBCA by $13.2 \%$, GLBCA by $18 \%$, LDC by $20.4 \%$, and PSO-C by $23.4 \%$.

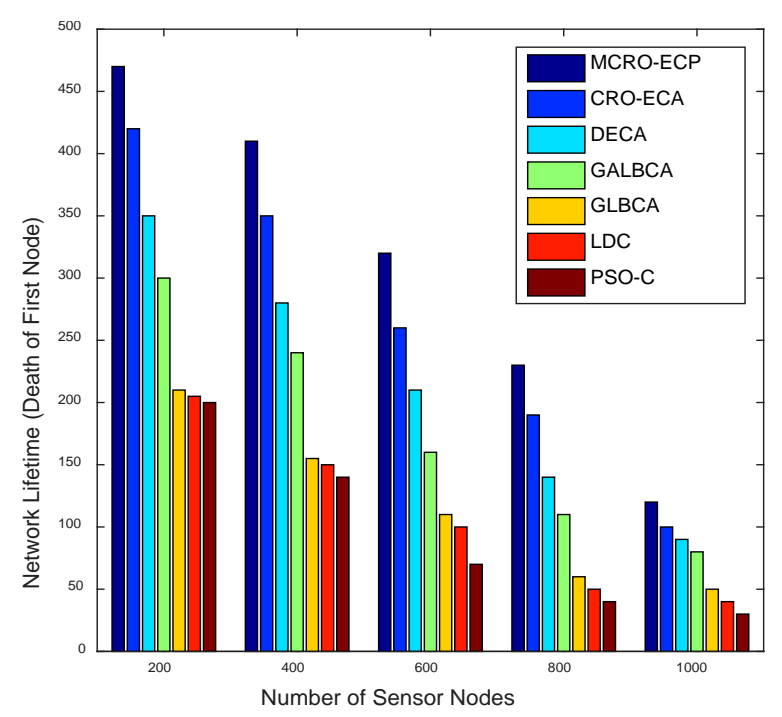

Fig. 3. Performance analysis of network lifetime in 400 sensor nodes and 40 cluster heads wireless sensor network.

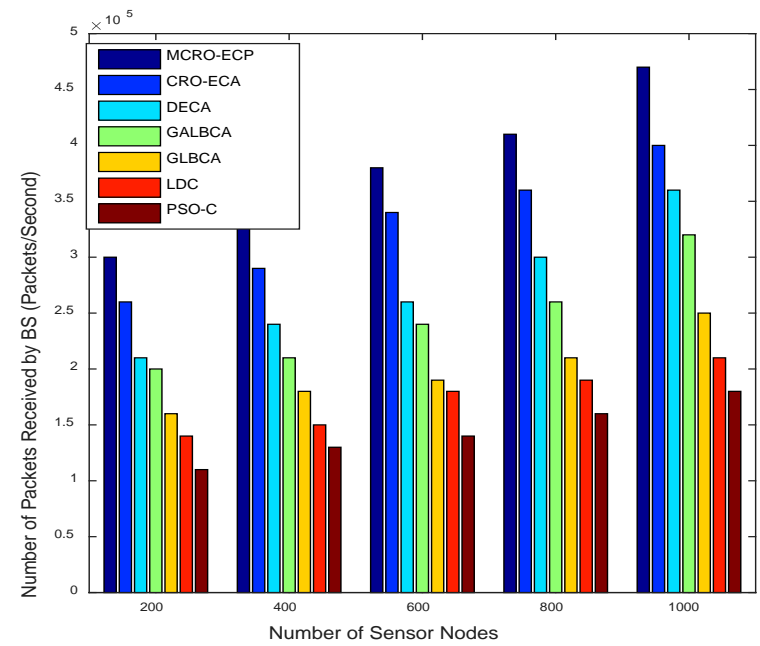

Fig. 4. Performance analysis of packets receipt by base station in 400 sensor nodes and 40 cluster heads wireless sensor network.

D) Performance Analysis in terms of Convergence Rate

To converge the global optimal solution, an algorithm performs certain number of rounds 
called as convergence rate. Mutation chemical reaction algorithm is belong to iterative method. As iterative method, a fundamental question is their convergence rates, how fast does an MCRO converge to the optimum per generation?. Convergence rate measures the rate of the fitness change per round. The convergence rate of proposed protocol, MCRO-ECP was extensively tested. Fig. 5 proves better quality of solution provided by MCRO-ECP compared to other existing methods such as PSO-C, GALBCA, DECA and CRO-ECA protocols. Following the theoretical and experimental chemical kinetics, the faster convergence with enhanced quality of solution of MCRO-ECP is proven. According to the NFL (No Free Lunch) theorem, meta-heuristic is neither superior nor inferior to others. If a problem matches to a particular meta-heuristic then it out performs, i.e., MCRO-ECP out performs all other existing protocols.

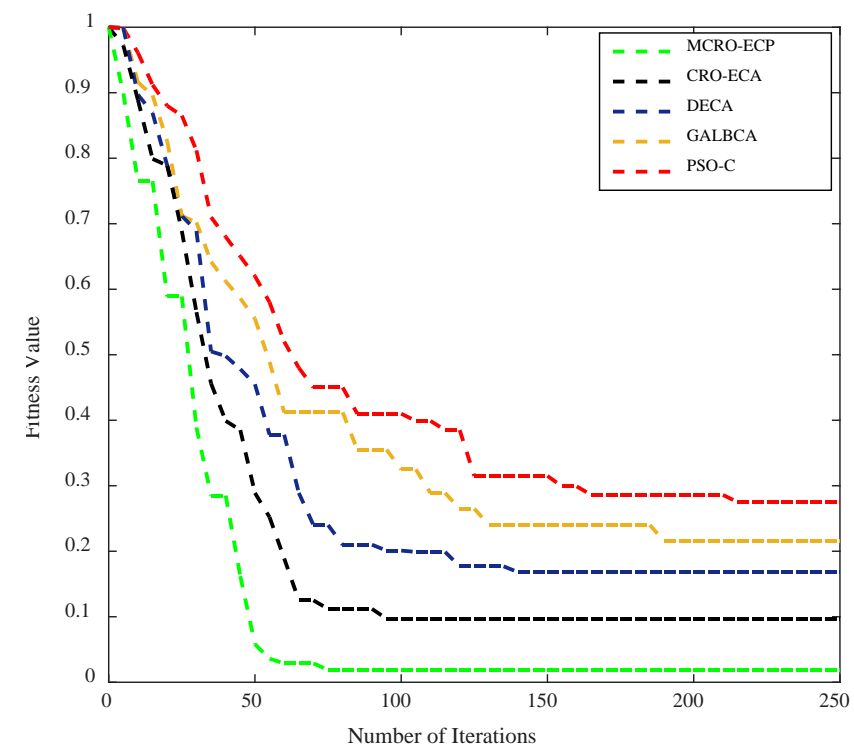

Fig. 5. Convergence rate analysis in 400 sensor nodes and 40 cluster heads wireless sensor network.

\section{Conclusion}

In this study, linear programming models have been designed and formulated for two optimization problems, i.e., $\mathrm{CH}$ selection and cluster formation. The proposed protocol has been presented for the same, based on a novel MCRO technique using efficient molecular structure representations and potential energy functions. For the selection of optimal $\mathrm{CH}$, the neighbour node distance, base station distance and residual energy of sensor nodes are used as key parameters. For cluster formation, intra communication distance and $\mathrm{CH}$ node degree were the chosen parameters. In the communication phase, TDMA was used for intra node communication and direct communication was used for inter node communication. Simulation results along with peer comparisons have been presented. The proposed protocol is tested extensively under different conditions as such varying the number of sensor nodes and the number of cluster heads. The experimental results have shown that the proposed MCRO-ECP protocol performs and produced better output, in terms of total energy consumption, network lifetime, number of data packets received by the base station and convergence rate, than the already existing protocols. Development of improved clustering and routing protocols adopting meta-heuristic approaches is the aim in the future. 


\section{References}

[1] Akyildiz, I.F., Su, W., Sankarasubramaniam, Y., Cayirci, E., "Wireless sensor networks: a survey," Comput. Networks, 38, 393-422, 2002. Article (CrossRef Link).

[2] Seo, Jae-Hyun \& Kim, Yong-Hyuk \& Ryou, Hwang-Bin \& Cha,Si-Ho \& Jo,Minho., "Optimal Sensor Deployment for Wireless Surveillance Sensor Networks by a Hybrid Steady-State Genetic Algorithm," IEICE Transactions on Communications, vol. E91-B, pp. 3534-3543, 2008. Article (CrossRef Link).

[3] Afsar, M. M., \& Tayarani-N, M. H., "Clustering in sensor networks: A literature survey,” Journal of Network and Computer Applications, 46, 198-226, 2014.

Article (CrossRef Link).

[4] Liu, X., "A survey on clustering routing protocols in wireless sensor networks," Sensors, 12(8), 11113-11153, 2012. Article (CrossRef Link).

[5] D. E. Goldberg, "Genetic Protocols in Search, Optimization and Machine Learning," Addison-Wesley Longman, Boston, Mass, USA, 1989.

[6] J. H. Holland, "Adaptation in Natural and Artificial Systems: An Introductory Analysis with Applications to Biology, Control and Artificial Intelligence," MIT Press, Cambridge, Mass, USA, 1992.

[7] J. Kennedy and R. Eberhart, "Particle swarm optimization," in Proc. of the IEEE International Conference on Neural Networks, vol. 4, pp. 1942-1948, 1995. Article (CrossRef Link).

[8] M.Dorigo,V.Maniezzo, and A. Colorni, "Ant system: optimization by a colony of cooperating agents," IEEE Transactions on Systems, Man, and Cybernetics, Part B: Cybernetics, vol. 26, no. 1, pp. 29-41, 1996. Article (CrossRef Link).

[9] W.F. Gao, S.Y. Liu, and L.L. Huang, "A novel artificial bee colony protocol based on modified search equation and orthogonal learning," IEEE Transactions on Cybernetics, vol. 43, no. 3, pp. 1011-1024, 2013. Article (CrossRef Link).

[10] Z.W. Geem, J. H. Kim, and G. V. Loganathan, "A new heuristic optimization protocol: harmony search,” Simulation, vol. 76, no. 2, pp. 60-68, 2001. Article (CrossRef Link).

[11] Y. S. Lam and V. O. K. Li, "Chemical-reaction-inspired metaheuristic for optimization,” IEEE Transactions on Evolutionary Computation, vol. 14, no. 3, pp. 381-399, 2010.

Article (CrossRef Link).

[12] Y. S. Lam, V. O. K. Li, and J. J. Q. Yu, "Real-coded chemical reaction optimization,” IEEE Transactions on Evolutionary Computation, vol. 16, no. 3, pp. 339-353, 2012. Article (CrossRef Link).

[13] T.T.Nguyen, Z. Y. Li, S.W.Zhang, and T. K. Truong, "A protocol based on particle swarm and chemical reaction optimization," Expert Systems with Applications, vol. 41, no. 5, pp. 2134-2143, 2014. Article (CrossRef Link).

[14] J. J.Q. Yu, A. Y. S. Lam, and V.O. K. Li, "Evolutionary artificial neural network based on chemical reaction optimization," in Proc. of the IEEE Congress on Evolutionary Computation (CEC '11), IEEE, New Orleans, La, USA, pp. 2083-2090, 2011. Article (CrossRef Link).

[15] Ransikarn Ngambusabongsopa, Zhiyong Li, and Esraa Eldesouky, "A Hybrid Mutation Chemical Reaction Optimization Algorithm for Global Numerical Optimization,” Mathematical Problems in Engineering, vol. 2015, Article ID 375902, p.17, 2015. Article (CrossRef Link).

[16] D. W. Oxtoby, H. P. Gill, A., “Campion. Principles of modern chemistry,” 7th Edition, Cengage Learning, Unit 3 and Unit 5, 2012.

[17] Heinzelman, W. R., Chandrakasan, A., \& Balakrishnan, H., "Energy-efficient communication protocol for wireless microsensor networks," in Proc. of InSystem sciences-2000, IEEE Proceedings of the 33rd annual Hawaii international conference on, 2000.

Article (CrossRef Link). 
[18] Ravuri Daniel and Kuda Nageswara Rao, “An Optimal Power Conservation Cluster based Routing Algorithm using Fuzzy Verdict Mechanism for Wireless Sensor Networks,” in Proc. of IEEE Conference on Electrical, Electronics, Signals, Communication \& Optimization-EESCO, 2015. Article (CrossRef Link).

[19] Younis, O., \& Fahmy, S., "HEED: energy efficient distributed clustering approach for Ad-Hoc sensor networks,” IEEE Transactions on Mobile Computing, 3(4), 366-379, 2004.

Article (CrossRef Link).

[20] Bandyopadhyay, S., \& Coyle, E. J., “An energy efficient hierarchical clustering protocol for wireless sensor networks,” IEEE INFOCOMM, Vol. 3, pp. 1713-1723, 2003.

[21] Banerjee, S., \& Khuller, S., A, “clustering scheme for hierarchical control in wireless networks,” in Proc. of IEEE INFOCOMM, Vol. 2, pp. 1028-1037, 2001.

[22] Bari, A., Jaekel, A., \& Bandyopadhyay, S., "Clustering strategies for improving the lifetime of two-tiered sensor networks,” Computer Communications, 31(14), 3451-3459, 2008. Article (CrossRef Link).

[23] Low, C. P., Fang, C., Ng, J. M., \& Ang, Y. H., "Efficient load-balanced clustering protocols for wireless sensor networks,” Computer Communications, 31(4), 750-759, 2008.

Article (CrossRef Link).

[24] Heinzelman, W.B., Chandrakasan, A.P., Balakrishnan, H., “An application specific protocol architecture for wireless micro-sensor networks,” IEEE Trans. Wirel. Commun, 1(4), 660-670, 2002. Article (CrossRef Link).

[25] Tillet, J., Rao, R., \& Sachin, F., “Cluster head identification in ad-hoc sensor networks using particle swarm optimization,” in Proc. of IEEE international conference on personal wireless communications, pp. 201-205, 2002. Article (CrossRef Link).

[26] Enan, A., Bara, A., \& Attea, A., "Energy-aware evolutionary routing protocol for dynamic clustering of wireless sensor networks," Swarm and Evolutionary Computation, 1(4), 195-203, 2011. Article (CrossRef Link).

[27] P.C Srinivasa Rao, P.K Jana, and Haider Banka, "A particle swarm optimization based energy efficient cluster head selection algorithm for wireless sensor networks," Wireless networks, Volume 23, Issue 7, Pages 2005-2020, 2017.

[28] Latiff, N. M. A., Tsemenidis, C. C., \& Sheriff, B. S., "Energy-aware clustering for wireless sensor networks using particle swarm optimization," in Proc. of 18th annual IEEE international symposium on personal, indoor and mobile radio communications, pp. 1-5, 2007.

Article (CrossRef Link).

[29] Buddha, S., \& Lobiyal, D. K., “A novel energy-aware cluster head selection based on particle swarm optimization for wireless sensor networks,” Human-Centric Computing and Information Sciences, 2(1), 2-13, 2012.

[30] Kuila, P., Gupta, S. K., \& Jana, P. K., “A novel evolutionary approach for load balanced clustering problem for wireless sensor networks,” Swarm and Evolutionary Computation, 12, 48-56, 2013. Article (CrossRef Link).

[31] Kuila, P., \& Jana, P. K., “A novel differential evolution based clustering protocol for wireless sensor networks,” Applied Soft Computing, 25, 414-425, 2014. Article (CrossRef Link).

[32] Srinivasa Rao, P.C., Banka, H., "Energy efficient clustering protocols for wireless sensor networks: novel chemical reaction optimization approach,” Wireless Networks, Volume 23, Issue 2, pp 433-452, 2017. Article (CrossRef Link).

[33] E. Guggenheim, "Thermodynamics: An Advanced Treatment for Chemists and Physicists," Wiley, NorthHolland, 5th edition, 1967.

[34] Xu, J., Liu, W., Lang, F., Zhang, Y., \& Wang, C., "Distance measurement model based on RSSI in WSN,” Wireless Sensor Networks, 2(8), 606-611, 2010. Article (CrossRef Link).

[35] Dietrich, I., \& Dressler, F., “On the lifetime of wireless sensor networks,” ACM Transactions on Sensor Networks, 5(1), 1-38, 2009. Article (CrossRef Link). 


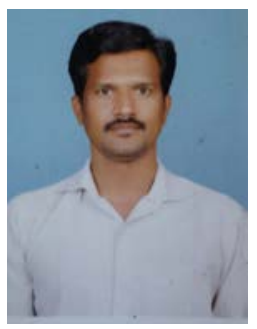

Ravuri Daniel, research scholar in the Department of Computer Science and Engineering, JNTUK-Kakinada, Indian. His interest of research includes wireless sensor networks, computer networks, IoT and nature inspired optimization techniques.

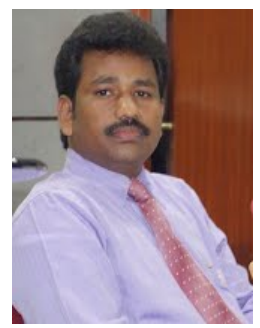

Dr.Kuda Nageswara Rao, Professor, Head of the department of Computer Science \& Systems Engineering, Andhra University College of Engineering (A), Andhra University, Visakhapatnam, India. His research interests includes Computer Networks, IoT, TCP/IP, Internet Technologies, Wireless and Wireless Sensor Networks. Life member in ISPS and ISTE, member in IETE, ISCA, CSI etc., 